\title{
eNOS polymorphisms and moyamoya disease
}

\author{
Young Seok Park • Nam Keun Kim
}

Received: 27 July 2011 / Accepted: 29 July 2011 / Published online: 11 August 2011

(C) Springer-Verlag 2011

The moyamoya disease shows various clinical features. It is difficult to identify as specific genes associated with it are not identified yet. Although progress in the search for genetic loci underlying moyamoya disease is encouraging, a relevant specific single gene has not yet been identified.
Moyamoya disease appears to be a multifactorial, polygenic disorder that does not display a classic pattern of inheritance. eNOS may be just one candidate gene among many that influence the clinical features which characterize the disease.

Y. S. Park $(\bowtie)$

Neurosurgery Department, Bundang CHA Medical Center,

CHA University,

Yatapdong 351, Bundanggu,

Seongnam 463-712, South Korea

e-mail: yspark7@cha.ac.kr

N. K. Kim

Institute for Clinical Research, CHA University,

Seongnam, South Korea 